University of South Florida

DIGITAL COMMONS Digital Commons @ University of @ UNIVERSITY OF SOUTH FLORIDA South Florida

$1-1-2016$

\title{
2016 Work Plan USF Sarasota-Manatee
}

USF

Follow this and additional works at: https://digitalcommons.usf.edu/usf_accountability_reports

\section{Scholar Commons Citation}

USF, "2016 Work Plan USF Sarasota-Manatee" (2016). USF Accountability Reports. 61.

https://digitalcommons.usf.edu/usf_accountability_reports/61

This Article is brought to you for free and open access by the USF Archives at Digital Commons @ University of South Florida. It has been accepted for inclusion in USF Accountability Reports by an authorized administrator of Digital Commons @ University of South Florida. For more information, please contact digitalcommons@usf.edu. 


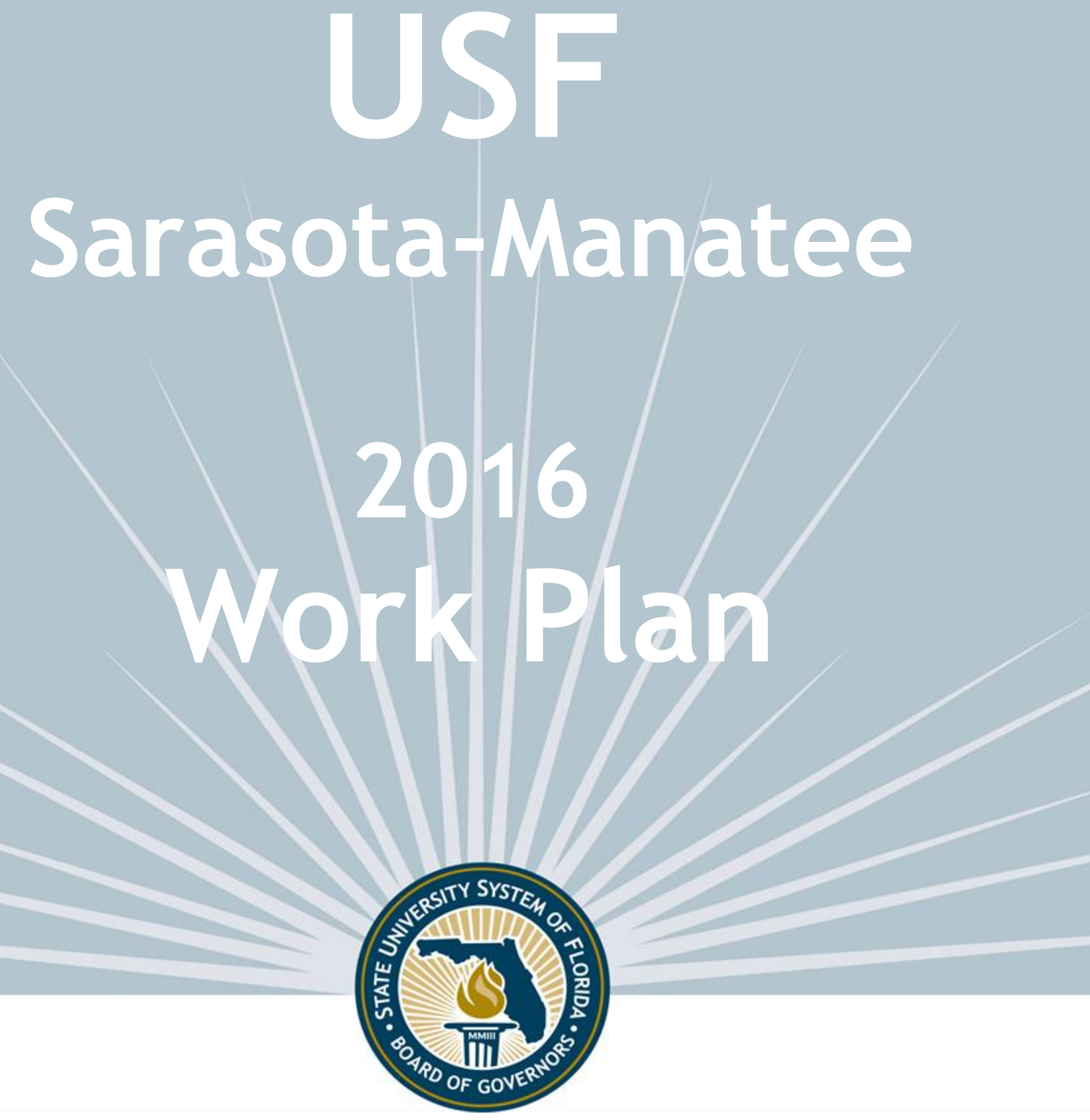

University of South Florida-Sarasota-Manatee

University Work Plan Presentation

for Board of Governors June 2016 Meeting

STATE UNIVERSITY SYSTEM of FLORIDA $\mid$ Board of Governors 


\section{INTRODUCTION}

The State University System of Florida has developed three tools that aid in guiding the System's future.

1) The Board of Governors' 2025 System Strategic Plan is driven by goals and associated metrics that stake out where the System is headed;

2) The Board's Annual Accountability Report provides yearly tracking for how the System is progressing toward its goals;

3) Institutional Work Plans connect the two and create an opportunity for greater dialogue relative to how each institution contributes to the System's overall vision.

These three documents assist the Board with strategic planning and with setting short-, mid-and long-term goals. The Board will use these documents to help advocate for all System institutions and foster even greater coordination with the institutions and their Boards of Trustees.

Longer-term goals will inform future agendas of the Board's Strategic Planning Committee. The Board's acceptance of a work plan does not constitute approval of any particular component, nor does it supersede any necessary approval processes that may be required for each component. 


\section{TABLE OF CONTENTS}

1. STRATEGY
a. Mission Statement
b. Vision Statement
c. Statement of Strategy
d. Strengths and Opportunities
e. Key Initiatives \& Investments

2. PERFORMANCE BASED FUNDING METRICS

3. KEY PERFORMANCE INDICATORS

a. Teaching \& Learning

b. Institution Specific Goals

4. ENROLLMENT PLANNING

5. ACADEMIC PROGRAM COORDINATION

6. STUDENT DEBT \& NET COST

7. UNIVERSITY REVENUES

8. TUITION, FEES AND HOUSING PROJECTIONS

9. DEFINITIONS 


\section{MISSION STATEMENT (What is your purpose?)}

The University of South Florida Sarasota-Manatee provides high quality bachelor's and graduate-level education and scholarly activity in a personalized learning community that prepares successful leaders and responsible citizens.

\section{VISION STATEMENT (What do you aspire to?)}

As a valued member of the USF System, the University of South Florida Sarasota-Manatee will be nationally recognized as a student-centered, research-focused, community-engaged university with significant economic and cultural impact to the region.

\section{STATEMENT OF STRATEGY (How will you get there?)}

Given your mission, vision, strengths and available resources, provide a brief description of your market and your strategy for addressing and leading it.

USF Sarasota-Manatee's primary focus is to provide quality, affordable, bachelor's and master's degrees that address the specific higher education needs of the region. USF Sarasota-Manatee serves its market by offering a mix of online and classroom instruction at times that are convenient to a commuter student population. USF Sarasota-Manatee also partners with local businesses, non-profits, and educational institutions to ensure the institution is meeting the need for quality internship opportunities for students and producing talented graduates to meet the needs of the local workforce. 


\section{STRENGTHS AND OPPORTUNITIES (within 3 years)}

\section{What are your core capabilities, opportunities and challenges for improvement?}

USF Sarasota-Manatee provides access to a personalized, learning experience that permits students to "learn where they earn." The institution capitalizes on its low student-to-faculty ratio by offering individualized attention to ensure that students are supported, retained and successful. As a member of the USF System, USF Sarasota-Manatee benefits from the efficiency of shared resources, a unified brand that yields identity and impact, and the opportunity for collaboration with other USF System institutions including via tailored 2+2 programs to meet the needs of the Tampa Bay region without unnecessary duplication of programs.

One such example of this is a burgeoning new partnership between USF and USF Sarasota-Manatee in engineering, where USF Sarasota-Manatee students will complete two years of pre-engineering course work on their home campus, complete an AA certificate and then transfer to the USF campus to obtain a baccalaureate in engineering. This allows the USF System to serve the growing workforce need for more engineers without building another costly engineering program across the Bay. USF Sarasota-Manatee will continue to seek out similar partnerships across the USF System and with its other community partners to increase its profile and serve its mission as a regional institution dedicated to supporting the local knowledge economy.

\section{KEY INITIATIVES \& INVESTMENTS (within 3 years)}

Describe your top three key initiatives for the next three years that will drive improvement in Academic Quality, Operational Efficiency, and Return on Investment.

\section{Reach the goals of the USF Sarasota-Manatee 2015-20 Strategic Plan, Focus on Quality 2020:}

USF Sarasota-Manatee faculty, staff, students, alumni, Campus Board members, and community representatives selected seven major strategic goals as part of the new Strategic Plan, which aligns with the USF System's guiding principles and SUS goals: (1) Support Student Success, (2) Enhance Campus Life, (3) Engage in Intentional Enrollment Management, (4) Strengthen Teaching \& Learning, (5) Promote Scholarly Activity, (6) Maximize Community Engagement, and (7) Cultivate Resources. Fiftyfive specific strategies are being implemented to meet the goals of the Strategic Plan. As of the first sixmonth update, USF Sarasota-Manatee made progress on all but two of these strategies.

\section{Expand STEM and Areas of Strategic Emphasis offerings:}

USF Sarasota-Manatee's College of Science and Mathematics opens in Fall 2016 with a founding dean, who will align applied science and mathematics offerings on the campus. The College will pursue the following key initiatives: (1) a $2+2$ engineering program in partnership with College of Engineering on the USF campus in Tampa, (2) expansion of laboratory and research space, and (3) enrollment growth in STEM and Areas of Strategic Emphasis programs.

\section{Preserve, grow, and diversify USF Sarasota-Manatee resources:}

USF Sarasota-Manatee will strategically increase efforts in grant-writing, private fundraising, and public-private partnership development to maximize its resources in order to invest in those areas that will help it advance its Strategic Plan goals, performance-based funding metrics and other USF System opportunities for advancement. 


\section{PERFORMANCE BASED FUNDING METRICS}

\begin{tabular}{|c|c|c|c|c|c|c|}
\hline & $\begin{array}{r}2015 \\
\text { ACTUAL } \\
\end{array}$ & $\begin{array}{c}2016 \\
\text { ACTUAL } \\
\end{array}$ & $\begin{array}{l}2017 \\
\text { GOALS }\end{array}$ & $\begin{array}{r}2018 \\
\text { GOALS }\end{array}$ & $\begin{array}{r}2019 \\
\text { GOALS }\end{array}$ & $\begin{array}{r}2020 \\
\text { GOALS } \\
\end{array}$ \\
\hline $\begin{array}{l}\text { Percent of Bachelor's Graduates } \\
\text { Enrolled or Employed }(\mathbf{\$ 2 5 , 0 0 0 + )} \\
\text { within the U.S. One Year After Graduation }\end{array}$ & $\begin{array}{c}65.5 \% \\
2012-13\end{array}$ & $\begin{array}{l}62.8 \% \\
2013-14\end{array}$ & $\begin{array}{c}63.5 \% \\
2014-15\end{array}$ & $\begin{array}{c}66.0 \% \\
2015-16\end{array}$ & $\begin{array}{l}68.0 \% \\
2016-17\end{array}$ & $\begin{array}{c}70.0 \% \\
2017-18\end{array}$ \\
\hline $\begin{array}{l}\text { Median Wages of Bachelor's } \\
\text { Graduates Employed Full-time } \\
\text { in Florida One-Year After Graduation }\end{array}$ & $\underset{2012-13}{\$ 36,000}$ & $\begin{array}{l}\$ 35,600 \\
2013-14\end{array}$ & $\begin{array}{l}\$ 36,000 \\
2014-15\end{array}$ & $\begin{array}{c}\$ 36,500 \\
2015-16\end{array}$ & $\begin{array}{c}\$ 37,000 \\
2016-17\end{array}$ & $\begin{array}{c}\$ 37,500 \\
2017-18\end{array}$ \\
\hline $\begin{array}{l}\text { Cost per Bachelor's Degree } \\
\text { Costs to the University }\end{array}$ & \multicolumn{6}{|c|}{ Data reported at the USF System level only. } \\
\hline $\begin{array}{l}\text { FTIC } 6 \text { year Graduation Rate } \\
\text { for full- and part-time students }\end{array}$ & $\underset{2008-14}{\dot{2}}$ & $\dot{2009-15}$ & $\stackrel{\cdot}{2010-16}$ & $\dot{\cdot}$ & $\dot{\cdot}$ & $\begin{array}{c}59.0 \% \\
2013-19\end{array}$ \\
\hline $\begin{array}{l}\text { Academic Progress Rate } * \\
\text { FTIC } 2 \text { year Retention Rate with GPA>2 }\end{array}$ & $\begin{array}{c}69.9 \%^{1} \\
2013-14\end{array}$ & $\begin{array}{c}78.7 \%^{2} \\
2014-15\end{array}$ & $\begin{array}{c}80.2 \% \\
2015-16\end{array}$ & $\begin{array}{c}82.0 \% \\
2016-17\end{array}$ & $\begin{array}{l}84.0 \% \\
2017-18\end{array}$ & $\begin{array}{c}86.0 \% \\
2018-19\end{array}$ \\
\hline $\begin{array}{l}\text { Bachelor's Degrees Awarded Within } \\
\text { Programs of Strategic Emphasis }\end{array}$ & $\begin{array}{c}33.9 \% \\
2013-14\end{array}$ & $\begin{array}{c}41.1 \% \\
2014-15\end{array}$ & $\begin{array}{c}42.0 \% \\
2015-16\end{array}$ & $\begin{array}{c}43.0 \% \\
2016-17\end{array}$ & $\begin{array}{c}44.0 \% \\
2017-18\end{array}$ & $\begin{array}{c}45.0 \% \\
2018-19\end{array}$ \\
\hline $\begin{array}{l}\text { University Access Rate } \\
\text { Percent of Fall Undergraduates } \\
\text { with a Pell grant }\end{array}$ & $\begin{array}{l}40.7 \% \\
\text { Fall } 2013\end{array}$ & $\begin{array}{l}41.0 \% \\
\text { Fall } 2014\end{array}$ & $\begin{array}{l}41.0 \% \\
\text { Fall } 2015\end{array}$ & $\begin{array}{l}41.0 \% \\
\text { Fall } 2016\end{array}$ & $\begin{array}{l}41.0 \% \\
\text { Fall } 2017\end{array}$ & $\begin{array}{l}41.0 \% \\
\text { Fall } 2018\end{array}$ \\
\hline $\begin{array}{l}\text { Graduate Degrees Awarded Within } \\
\text { Programs of Strategic Emphasis }\end{array}$ & $\begin{array}{c}27.3 \% \\
2013-14\end{array}$ & $\begin{array}{c}12.0 \% \\
2014-15\end{array}$ & $\begin{array}{c}19.0 \% \\
2015-16\end{array}$ & $\begin{array}{c}19.5 \% \\
2016-17\end{array}$ & $\begin{array}{c}20.0 \% \\
2017-18\end{array}$ & $\begin{array}{c}20.5 \% \\
2018-19\end{array}$ \\
\hline $\begin{array}{l}\text { BOG METRIC: } \\
\text { Percent of Bachelor's Degrees } \\
\text { Without Excess Hours }\end{array}$ & $\begin{array}{c}75.4 \% \\
2013-14\end{array}$ & $\begin{array}{c}71.7 \% \\
2014-15\end{array}$ & $\begin{array}{c}72.9 \% \\
2015-16\end{array}$ & $\begin{array}{c}76.0 \% \\
2016-17\end{array}$ & $\begin{array}{c}78.0 \% \\
2017-18\end{array}$ & $\begin{array}{c}80.0 \% \\
2018-19\end{array}$ \\
\hline $\begin{array}{l}\text { UBOT METRIC: } \\
\text { Number of Post-doctoral } \\
\text { Appointees }\end{array}$ & $\begin{array}{c}0 \\
\text { Fall } 2012\end{array}$ & $\begin{array}{c}0 \\
\text { Fall } 2013\end{array}$ & $\begin{array}{c}0 \\
\text { Fall } 2014\end{array}$ & $\begin{array}{c}5 \\
\text { Fall } 2015\end{array}$ & $\begin{array}{c}5 \\
\text { Fall } 2016\end{array}$ & $\begin{array}{c}5 \\
\text { Fall } 2017\end{array}$ \\
\hline
\end{tabular}

Note: Metrics are defined in appendix. For more information about the PBF model visit: http://www.flbog.edu/about/budget/performance funding.php. ${ }^{*} \mathrm{FTIC}$ retention/academic progress rate percentages represent students starting and ending at USFSM.

$177.1 \%$ of USFSM students of the 2013 cohort were retained within the USF System

$286.7 \%$ of USFSM students of the 2014 cohort were retained within the USF System 
Teaching \& Learning Metrics (from 2025 System Strategic Plan that are not included in PBF or Preeminence)

\begin{tabular}{|c|c|c|c|c|c|c|}
\hline & $\begin{array}{c}2015 \\
\text { ACTUAL }\end{array}$ & $\begin{array}{c}2016 \\
\text { ACTUAL }\end{array}$ & $\begin{array}{c}2017 \\
\text { GOALS }\end{array}$ & $\begin{array}{c}2018 \\
\text { GOALS }\end{array}$ & $\begin{array}{c}2019 \\
\text { GOALS }\end{array}$ & $\begin{array}{c}2020 \\
\text { GOALS }\end{array}$ \\
\hline $\begin{array}{l}\text { 2. Freshmen in Top } 10 \% \\
\text { of Graduating High School Class }\end{array}$ & $\begin{array}{l}29.0 \% \\
\text { Fall } 2014\end{array}$ & $\begin{array}{l}26.0 \% \\
\text { Fall } 2015\end{array}$ & $\begin{array}{l}26.0 \% \\
\text { Fall } 2016\end{array}$ & $\begin{array}{c}27.0 \% \\
\text { Fall } 2017\end{array}$ & $\begin{array}{c}28.0 \% \\
\text { Fall } 2018\end{array}$ & $\begin{array}{c}29.0 \% \\
\text { Fall } 2019\end{array}$ \\
\hline $\begin{array}{l}\text { 4. Time to Degree } \\
\text { for FTICs in 12Ohr programs }\end{array}$ & $\underset{2013-14}{\cdot}$ & 2014-15 & $\underset{2015-16}{\cdot}$ & $\begin{array}{c}4.2 \\
2016-17\end{array}$ & $\begin{array}{c}4.2 \\
2017-18\end{array}$ & $\begin{array}{c}4.2 \\
2018-19\end{array}$ \\
\hline $\begin{array}{l}\text { 5. Four-Year FTIC } \\
\text { Graduation Rates } \\
\text { full- and part-time students }\end{array}$ & $2010-14$ & 2011-15 & 2012-16 & $\begin{array}{l}35.0 \% \\
2013-17\end{array}$ & $\begin{array}{c}36.0 \% \\
2014-18\end{array}$ & $\begin{array}{c}37.0 \% \\
2015-19\end{array}$ \\
\hline $\begin{array}{l}\text { 8. Bachelor's Degrees Awarded } \\
\text { First Majors Only }\end{array}$ & $\begin{array}{l}490 \\
2013-14\end{array}$ & $\begin{array}{l}474 \\
2014-15\end{array}$ & $\begin{array}{l}495 \\
2015-16\end{array}$ & $\begin{array}{c}512 \\
2016-17\end{array}$ & $\begin{array}{c}530 \\
2017-18\end{array}$ & $\begin{array}{c}549 \\
2018-19\end{array}$ \\
\hline $\begin{array}{l}\text { 9. Graduate Degrees Awarded } \\
\text { First Majors Only }\end{array}$ & $\begin{array}{c}55 \\
2013-14\end{array}$ & $\begin{array}{c}50 \\
2014-15\end{array}$ & $\begin{array}{c}52 \\
2015-16\end{array}$ & $\begin{array}{c}54 \\
2016-17\end{array}$ & $\begin{array}{c}55 \\
2017-18\end{array}$ & $\begin{array}{c}57 \\
2018-19\end{array}$ \\
\hline $\begin{array}{l}\text { 10. Bachelor's Degrees Awarded } \\
\text { to African-American \& Hispanic } \\
\text { Students }\end{array}$ & $\begin{array}{c}15.0 \% \\
2013-14\end{array}$ & $\begin{array}{c}23.0 \% \\
2014-15\end{array}$ & $\begin{array}{c}23.0 \% \\
2015-16\end{array}$ & $\begin{array}{c}24.0 \% \\
2016-17\end{array}$ & $\begin{array}{c}25.1 \% \\
2017-18\end{array}$ & $\begin{array}{c}26.0 \% \\
2018-19\end{array}$ \\
\hline $\begin{array}{l}\text { 11. Adult (Aged } 25+\text { ) } \\
\text { Undergraduates Enrolled }\end{array}$ & $\begin{array}{l}57.2 \% \\
\text { Fall } 2013\end{array}$ & $\begin{array}{l}53.1 \% \\
\text { Fall } 2014\end{array}$ & $\begin{array}{l}49.7 \% \\
\text { Fall } 2015\end{array}$ & $\begin{array}{l}49.0 \% \\
\text { Fall } 2016\end{array}$ & $\begin{array}{l}48.0 \% \\
\text { Fall } 2017\end{array}$ & $\begin{array}{l}47.0 \% \\
\text { Fall } 2018\end{array}$ \\
\hline $\begin{array}{l}\text { 12. Percent of Undergraduate } \\
\text { FTE in Online Courses }\end{array}$ & $\begin{array}{l}49.9 \% \\
2013-14\end{array}$ & $\begin{array}{c}48.9 \% \\
2014-15\end{array}$ & $\begin{array}{l}52.4 \% \\
2015-16\end{array}$ & $\begin{array}{c}52.7 \% \\
2016-17\end{array}$ & $\begin{array}{c}52.9 \% \\
2017-18\end{array}$ & $\begin{array}{c}53.1 \% \\
2018-19\end{array}$ \\
\hline $\begin{array}{l}\text { 16. Percent of Bachelor's } \\
\text { Degrees in STEM \& Health }\end{array}$ & $\begin{array}{l}13.9 \% \\
2013-14\end{array}$ & $\begin{array}{l}18.8 \% \\
2014-15\end{array}$ & $\begin{array}{c}20.0 \% \\
2015-16\end{array}$ & $\begin{array}{c}21.1 \% \\
2016-17\end{array}$ & $\begin{array}{c}22.1 \% \\
2017-18\end{array}$ & $\begin{array}{c}23.0 \% \\
2018-19\end{array}$ \\
\hline $\begin{array}{l}\text { 18. Percent of Graduate Degrees } \\
\text { in STEM \& Health }\end{array}$ & $\begin{array}{c}0 \% \\
2013-14\end{array}$ & $\begin{array}{c}0 \% \\
2014-15\end{array}$ & $\begin{array}{c}0 \% \\
2015-16\end{array}$ & $\begin{array}{c}0 \% \\
2016-17\end{array}$ & $\begin{array}{c}0 \% \\
2017-18\end{array}$ & $\begin{array}{c}0 \% \\
2018-19\end{array}$ \\
\hline IMPROVING METRICS & & $\begin{array}{c}3 \\
\text { of } 8\end{array}$ & $\begin{array}{c}5 \\
\text { of } 8\end{array}$ & $\begin{array}{c}7 \\
\text { of } 8\end{array}$ & $\begin{array}{c}8 \\
\text { of } 10\end{array}$ & $\begin{array}{c}8 \\
\text { of } 10\end{array}$ \\
\hline
\end{tabular}




\section{Institution Specific Goals (optional)}

To further distinguish the university's distinctive mission, the university may choose to provide additional narrative and metric goals that are based on the university's own strategic plan.

\section{Narrative Goals.}

USF Sarasota-Manatee will submit an application to receive the Elective Community Engagement Classification in Spring 2019. According to the Carnegie Foundation Timeline, designation will be announced in January 2020.

\begin{tabular}{lcc|cccc} 
& 2015 & 2016 & 2017 & 2018 & 2019 & 2020 \\
& ACTUAL & ACTUAL & GOALS & GOALS & GOALS & GOALS \\
\hline $\begin{array}{l}\text { Metric \#1: } \\
\text { \% of Non-White Faculty \& Staff }\end{array}$ & $\begin{array}{c}18 \% \\
\text { Fall 2014 }\end{array}$ & $\begin{array}{c}19 \% \\
\text { Fall 2015 }\end{array}$ & $\begin{array}{c}19 \% \\
\text { Fall 2016 }\end{array}$ & $\begin{array}{l}20 \% \\
\text { Fall 2017 }\end{array}$ & $\begin{array}{l}20 \% \\
\text { Fall 2018 }\end{array}$ & $21 \%$ \\
Fall 2019 \\
\hline $\begin{array}{l}\text { Metric \#2: } \\
\begin{array}{l}\text { \% of Hispanic \& African American } \\
\text { Students }\end{array}\end{array}$ & $\begin{array}{c}20 \% \\
\text { Fall 2014 }\end{array}$ & $\begin{array}{c}20 \% \\
\text { Fall 2015 }\end{array}$ & $\begin{array}{c}21 \% \\
\text { Fall 2016 }\end{array}$ & $\begin{array}{c}22 \% \\
\text { Fall 2017 }\end{array}$ & $\begin{array}{c}23 \% \\
\text { Fall 2018 }\end{array}$ & $\begin{array}{c}24 \% \\
\text { Fall 2019 }\end{array}$ \\
\hline
\end{tabular}


FINAL - JUNE 2016

\section{ENROLLMENT PLANNING}

Planned Headcount Enrollment by Student Type (for all students at all campuses)

\begin{tabular}{|c|c|c|c|c|c|c|c|}
\hline & $\begin{array}{c}\text { FALL } 2013 \\
\text { ACTUAL }\end{array}$ & $\begin{array}{l}\text { FALL } 2014 \\
\text { ACTUAL }\end{array}$ & $\begin{array}{c}\text { FALL } 2015 \\
\text { ACTUAL }\end{array}$ & $\begin{array}{l}\text { FALL } 2016 \\
\text { PLAN }\end{array}$ & $\begin{array}{l}\text { FALL } 2017 \\
\text { PLAN }\end{array}$ & $\begin{array}{l}\text { FALL } 2018 \\
\text { PLAN }\end{array}$ & $\begin{array}{l}\text { FALL } 2019 \\
\text { PLAN }\end{array}$ \\
\hline \multicolumn{8}{|l|}{ UNDERGRADUATE } \\
\hline FTIC & 109 & 196 & 271 & 280 & 290 & 300 & 311 \\
\hline AA Transfers ${ }^{1}$ & 995 & 944 & 940 & 808 & 837 & 866 & 896 \\
\hline Other ${ }^{2}$ & 567 & 552 & 546 & 740 & 766 & 793 & 820 \\
\hline Subtotal & 1,671 & 1,692 & 1,757 & 1,829 & 1,893 & 1,959 & 2,028 \\
\hline \multicolumn{8}{|l|}{ GRADUATE $^{3}$} \\
\hline Master's & 128 & 120 & 174 & 180 & 186 & 193 & 200 \\
\hline Research Doctoral & 0 & 0 & 0 & 0 & 0 & 0 & 0 \\
\hline Professional Doctoral & 0 & 0 & 0 & 0 & 0 & 0 & 0 \\
\hline Subtotal & 128 & 120 & 174 & 180 & 186 & 193 & 200 \\
\hline \multicolumn{8}{|l|}{ UNCLASSIFIED } \\
\hline H.S. Dual Enrolled & 0 & 0 & 0 & 0 & 0 & 0 & 0 \\
\hline Other ${ }^{4}$ & 88 & 105 & 113 & 107 & 110 & 114 & 118 \\
\hline Subtotal & 88 & 105 & 113 & 107 & 110 & 114 & 118 \\
\hline TOTAL & 1,887 & 1,917 & 2,044 & 2,116 & 2,190 & 2,266 & 2,346 \\
\hline
\end{tabular}

Notes: This table reports the number of students enrolled at the university by student type categories. The determination for undergraduate, graduate and unclassified is based on the institutional class level values. Unclassified refers to a student who has not yet been formally admitted into a degree program but is enrolled. The student type for undergraduates is based on the Type of Student at Time of Most Recent Admission. The student type for graduates is based on the degree that is sought and the student CIP code. (1) Includes AA Transfers from the Florida College System. (2) Undergraduate - Other includes Post-Baccalaureates who are seeking a degree. (3) Includes Medical students. (4) Unclassified - Other includes Post-Baccalaureates who are not seeking a degree.

Planned FTE Enrollment by Method of Instruction (for all students at all campuses)

\begin{tabular}{|c|c|c|c|c|c|c|c|}
\hline & $\begin{array}{l}2012-13 \\
\text { ACTUAL }\end{array}$ & $\begin{array}{l}2013-14 \\
\text { ACTUAL }\end{array}$ & $\begin{array}{l}2014-15 \\
\text { ACTUAL }\end{array}$ & $\begin{array}{c}2015-16 \\
\text { PLAN }\end{array}$ & $\begin{array}{c}2016-17 \\
\text { PLAN }\end{array}$ & $\begin{array}{c}2017-18 \\
\text { PLAN }\end{array}$ & $\begin{array}{c}2018-19 \\
\text { PLAN }\end{array}$ \\
\hline \multicolumn{8}{|l|}{ UNDERGRADUATE } \\
\hline Distance (80-100\%) & 725 & 722 & 743 & 875 & 919 & 965 & 1013 \\
\hline Hybrid (50-79\%) & 46 & 62 & 33 & 8 & 8 & 8 & 8 \\
\hline Traditional (0-50\%) & 709 & 663 & 743 & 787 & 818 & 852 & 885 \\
\hline Subtotal & 1,480 & 1,446 & 1,519 & 1,670 & 1,745 & 1,825 & 1,906 \\
\hline \multicolumn{8}{|l|}{ GRADUATE } \\
\hline Distance (80-100\%) & 30 & 32 & 44 & 51 & 54 & 56 & 59 \\
\hline Hybrid (50-79\%) & 5 & 6 & 6 & 2 & 2 & 2 & 2 \\
\hline Traditional (0-50\%) & 99 & 97 & 78 & 110 & 113 & 118 & 123 \\
\hline Subtotal & 134 & 135 & 128 & 163 & 169 & 176 & 184 \\
\hline
\end{tabular}

Note: Full-time Equivalent (FTE) student is a measure of instructional activity that is based on the number of credit hours that students enroll. FTE is based on the standard national definition, which divides undergraduate credit hours by 30 and graduate credit hours by 24 . Distance Learning is a course in which at least 80 percent of the direct instruction of the course is delivered using some form of technology when the student and instructor are separated by time or space, or both (per 1009.24(17), F.S.). Hybrid is a course where $50 \%$ to $79 \%$ of the instruction is delivered using some form of technology, when the student and instructor are separated by time or space, or both (per SUDS data element 2052). Traditional refers to primarily face to face instruction utilizing some form of technology for delivery of supplemental course materials for no more than $49 \%$ of instruction (per SUDS data element 2052).

Data Provided by USF at the institution level 


\section{ENROLLMENT PLANNING (continued)}

\section{Planned FTE Enrollment Plan by Student Level}

\begin{tabular}{|c|c|c|c|c|c|c|c|c|c|}
\hline & $\begin{array}{l}2014-15 \\
\text { ACTUAL }\end{array}$ & $\begin{array}{c}2015-16 \\
\text { ESTIMATE }\end{array}$ & $\begin{array}{c}2016-17 \\
\text { PLAN }\end{array}$ & $\begin{array}{c}2017-18 \\
\text { PLAN }\end{array}$ & $\begin{array}{c}2018-19 \\
\text { PLAN }\end{array}$ & $\begin{array}{c}2019-20 \\
\text { PLAN }\end{array}$ & $\begin{array}{c}2020-21 \\
\text { PLAN }\end{array}$ & $\begin{array}{c}2021-22 \\
\text { PLAN }\end{array}$ & $\begin{array}{c}\text { Annual } \\
\text { Growth } \\
\text { Rate }^{*}\end{array}$ \\
\hline \multicolumn{10}{|c|}{ STATE FUNDABLE } \\
\hline \multicolumn{10}{|l|}{ RESIDENT } \\
\hline LOWER & 338 & 393 & 410 & 429 & 448 & 468 & 489 & 511 & $4.5 \%$ \\
\hline UPPER & 1,072 & 1,157 & 1,210 & 1,264 & 1,321 & 1,380 & 1,442 & 1,507 & $4.5 \%$ \\
\hline GRAD I & 115 & 141 & 147 & 154 & 161 & 168 & 175 & 183 & $4.5 \%$ \\
\hline GRAD ॥ & 0 & 0 & 0 & 0 & 0 & 0 & 0 & 0 & $\mathrm{~N} / \mathrm{A}$ \\
\hline TOTAL & 1,525 & 1,691 & 1,767 & 1,846 & 1,930 & 2,016 & 2,107 & 2,202 & $4.5 \%$ \\
\hline
\end{tabular}

NON RESIDENT

\begin{tabular}{lrrrrrrrrr}
\hline LOWER & 22 & 32 & 33 & 35 & 36 & 38 & 39 & 41 & $4.5 \%$ \\
UPPER & 41 & 55 & 57 & 60 & 62 & 65 & 68 & 71 & $4.5 \%$ \\
GRAD I & 7 & 14 & 15 & 16 & 16 & 17 & 18 & 19 & $4.5 \%$ \\
GRAD II & 0 & 0 & 0 & 0 & 0 & 0 & 0 & 0 & N/A \\
\hline TOTAL & $\mathbf{7 1}$ & $\mathbf{1 0 1}$ & $\mathbf{1 0 5}$ & $\mathbf{1 1 0}$ & $\mathbf{1 1 5}$ & $\mathbf{1 2 0}$ & $\mathbf{1 2 6}$ & $\mathbf{1 3 1}$ & $\mathbf{4 . 5 \%}$
\end{tabular}

TOTAL

\begin{tabular}{|c|c|c|c|c|c|c|c|c|c|}
\hline LOWER & 361 & 424 & 443 & 463 & 484 & 506 & 529 & 553 & $4.5 \%$ \\
\hline UPPER & 1,113 & 1,212 & 1,267 & 1,324 & 1,383 & 1,445 & 1,510 & 1,578 & $4.5 \%$ \\
\hline GRAD I & 122 & 155 & 162 & 169 & 177 & 185 & 193 & 202 & $4.5 \%$ \\
\hline GRAD II & 0 & 0 & 0 & 0 & 0 & 0 & 0 & 0 & $\mathrm{~N} / \mathrm{A}$ \\
\hline TOTAL & 1,596 & 1,791 & 1,872 & 1,956 & 2,044 & 2,136 & 2,232 & 2,333 & $4.5 \%$ \\
\hline \multicolumn{10}{|c|}{ NOT STATE FUNDABLE } \\
\hline LOWER & 4 & 6 & 6 & 7 & 7 & 7 & 8 & 8 & $4.5 \%$ \\
\hline UPPER & 41 & 28 & 29 & 31 & 32 & 33 & 35 & 36 & $4.5 \%$ \\
\hline GRAD I & 6 & 7 & 7 & 7 & 8 & 8 & 8 & 9 & $4.5 \%$ \\
\hline GRAD II & 0 & 0 & 0 & 0 & 0 & 0 & 0 & 0 & $\mathrm{~N} / \mathrm{A}$ \\
\hline TOTAL & 51 & 41 & 42 & 44 & 46 & 48 & 51 & 53 & $4.5 \%$ \\
\hline
\end{tabular}

Note: Full-time Equivalent (FTE) student is a measure of instructional activity that is based on the number of credit hours that students enroll. FTE is based on the standard national definition, which divides undergraduate credit hours by 30 and graduate credit hours by 24 . Note*: The Planned Annual Growth Rate is a compounded rate based on the following formula: (2021-22 value divided by the 2016-17 value) to the (1/5) exponent minus one.

Data Provided by USF at the institution level 


\section{ACADEMIC PROGRAM COORDINATION}

New Programs For Consideration by University in AY 2016-17

The S.U.S. Council of Academic Vice Presidents (CAVP) Academic Program Coordination Work Group will review these programs as part of their on-going coordination efforts. The programs listed below are based on the 2015 Work Plan list for programs under consideration for 2016-17.

\begin{tabular}{|c|c|c|c|c|c|c|}
\hline PROGRAM TITLES & $\begin{array}{l}\text { CIP CODE } \\
\text { 6-digit }\end{array}$ & $\begin{array}{l}\text { AREA OF } \\
\text { STRATEGIC } \\
\text { EMPHASIS }\end{array}$ & $\begin{array}{c}\text { OTHER } \\
\text { UNIVERSITIES } \\
\text { WITH SAME } \\
\text { PROGRAM }\end{array}$ & $\begin{array}{l}\text { OFFERED VIA } \\
\text { DISTANCE } \\
\text { LEARNING } \\
\text { IN SYSTEM }\end{array}$ & $\begin{array}{l}\text { PROJECTED } \\
\text { ENROLLMENT } \\
\text { in 5th year }\end{array}$ & $\begin{array}{l}\text { PROPOSED } \\
\text { DATE OF } \\
\text { SUBMISSION } \\
\text { TO UBOT }\end{array}$ \\
\hline TRUGKAIVI IILLES & & & & & & \\
\hline
\end{tabular}

BACHELOR'S PROGRAMS

MASTER'S, SPECIALIST AND OTHER ADVANCED MASTER'S PROGRAMS

DOCTORAL PROGRAMS

New Programs For Consideration by University in 2017-19

These programs will be used in the 2017 Work Plan list for programs under consideration for 2017-18.

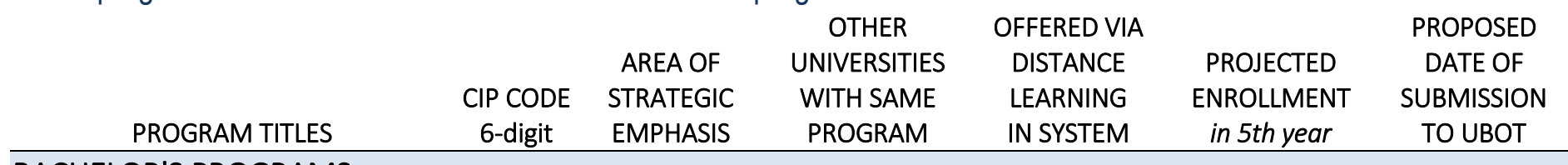

BACHELOR'S PROGRAMS

MASTER'S, SPECIALIST AND OTHER ADVANCED MASTER'S PROGRAMS

DOCTORAL PROGRAMS 
FINAL - JUNE 2016

\section{STUDENT DEBT \& NET COST}

\section{Student Debt Summary}

\begin{tabular}{lccccc} 
& $2010-11$ & $2011-12$ & $2012-13$ & $2013-14$ & $2014-15$ \\
\hline Percent of Bachelor's Recipients with Debt & $52 \%$ & $52 \%$ & $54 \%$ & $61 \%$ & $53 \%$ \\
\hline $\begin{array}{l}\text { Average Amount of Debt } \\
\text { for Bachelor's who have graduated with debt }\end{array}$ & $\$ 14,450$ & $\$ 17,790$ & $\$ 20,020$ & $\$ 16,350$ & $\$ 19,244$ \\
& & & & & $2012-15$ \\
NSLDS Cohort Year & $2008-11$ & $2009-12$ & $2010-13$ & $2011-14$ & Preliminary \\
\hline Student Loan Cohort Default Rate (3rd Year) & $10.1 \%$ & $9.8 \%$ & $7.5 \%$ & $5.4 \%$ & $5.2 \%$
\end{tabular}

Cost of Attendance (for Full-Time Undergraduate Florida Residents in the Fall and Spring of 2015-16)

\begin{tabular}{ccccccc} 
& $\begin{array}{c}\text { TUITION } \\
\& \text { FEES }\end{array}$ & $\begin{array}{c}\text { BOOKS } \& \\
\text { SUPPLIES }\end{array}$ & $\begin{array}{c}\text { ROOM } \\
\& \text { BOARD }\end{array}$ & TRANSPORTATION & $\begin{array}{c}\text { OTHER } \\
\text { EXPENSES }\end{array}$ & TOTAL \\
\hline AT HOME & $\$ 5,588$ & $\$ 1,200$ & $\$ 4,700$ & $\$ 1,600$ & $\$ 2,500$ & $\$ 15,588$ \\
\hline
\end{tabular}

Estimated Net Cost by Family Income (for Full-Time Undergraduate Florida Residents in the Fall and Spring of 2015-16)

\begin{tabular}{|c|c|c|c|c|c|c|}
\hline \multirow{2}{*}{$\begin{array}{c}\text { FAMILY } \\
\text { INCOME } \\
\text { GROUPS } \\
\end{array}$} & \multirow{2}{*}{\multicolumn{2}{|c|}{$\begin{array}{l}\text { FULL-TIME RESIDENT } \\
\text { UNDERGRADUATES }\end{array}$}} & \multirow{2}{*}{$\begin{array}{c}\text { AVG. NET } \\
\text { COST OF } \\
\text { ATTENDANCE }\end{array}$} & \multirow{2}{*}{$\begin{array}{l}\text { AVG. NET } \\
\text { TUITION } \\
\text { \& FEES } \\
\end{array}$} & \multirow{2}{*}{$\begin{array}{c}\text { AVG. } \\
\text { GIFT AID } \\
\text { AMOUNT }\end{array}$} & \multirow{2}{*}{$\begin{array}{c}\text { AVG. } \\
\text { LOAN } \\
\text { AMOUNT }\end{array}$} \\
\hline & & & & & & \\
\hline Below $\$ 40,000$ & 269 & $44 \%$ & $\$ 11,774$ & $-\$ 2,182$ & $\$ 7,075$ & $\$ 4,274$ \\
\hline$\$ 40,000-\$ 59,999$ & 64 & $11 \%$ & $\$ 12,004$ & $-\$ 1,195$ & $\$ 5,995$ & $\$ 2,300$ \\
\hline$\$ 60,000-\$ 79,999$ & 46 & $7 \%$ & $\$ 14,759$ & $\$ 1,325$ & $\$ 3,620$ & $\$ 3,202$ \\
\hline$\$ 80,000-\$ 99,999$ & 52 & $8 \%$ & $\$ 15,473$ & $\$ 2,449$ & $\$ 2,509$ & $\$ 3,137$ \\
\hline$\$ 100,000$ Above & 69 & $11 \%$ & $\$ 14,767$ & $\$ 2,767$ & $\$ 2,259$ & $\$ 2,827$ \\
\hline Not Reported & 114 & $19 \%$ & $\mathrm{n} / \mathrm{a}$ & $\$ 4,166$ & $\$ 782$ & $\$ 0$ \\
\hline TOTAL & 614 & $100 \%$ & $\$ 12,891^{*}$ & $\$ 311$ & $\$ 4,607$ & $\$ 2,936$ \\
\hline
\end{tabular}

Notes: This data only represents Fall and Spring financial aid data and is accurate as of March 31, 2016. Please note that small changes to Spring 2016 awards are possible before the data is finalized. Family Income Groups are based on the Total Family Income (including untaxed income) as reported on student FAFSA records. Full-time Students is a headcount based on at least 24 credit hours during Fall and Spring terms. Average Gift Aid includes all grants and scholarships from Federal, State, University and other private sources administered by the Financial Aid Office. Student waivers are also included in the Gift Aid amount. Gift Aid does not include the parental contribution towards EFC. Net Cost of Attendance is the actual average of the total Costs of Attendance (which will vary by income group due to the diversity of students living on- \& off- campus) minus the average Gift Aid amount. Net Tuition \& Fees is the actual average of the total costs of tuition and fees (which will vary by income group due to the amount of credit hours students are enrolled) minus the average Gift Aid amount (see page 16 for list of fees that are included). Average Loan Amount includes Federal (Perkins, Stafford, Ford Direct, and PLUS loans) and all private loans. 'Not Reported' represents the students who did not file a FAFSA. The bottom-line Total/Average represents the average of all full-time undergraduate Florida residents (note*: the total Net Cost of Attendance does not include students who did not report their family income data. 


\section{UNIVERSITY REVENUES}

University Revenues (in Millions of Dollars)

EDUCATION \& GENERAL

2014-15

2015-16

Main Operations

State Funds

$\$ 14.1$

$\$ 15.3$

Tuition

$\$ 8.0$

$\$ 9.0$

Subtotal

\$22.1

$\$ 24.9$

\section{OTHER BUDGET ENTITIES}

$\begin{array}{lcc}\text { Auxiliary Enterprises } & \$ 2.3 & \$ 2.2 \\ \text { Contracts \& Grants } & \$ 0.17 & \$ 0.6 \\ \text { Local Funds } & \$ 1.2 & \$ 1.1 \\ \text { Faculty Practice Plans } & \$ 0 & \$ 0\end{array}$

Note: State funds include recurring and non-recurring General Revenue funds, Lottery funds appropriated by the Florida Legislature. Actual tuition includes base tuition and tuition differential fee revenues for resident and nonresident undergraduate and graduate students net of waivers. Source: Tables $1 \mathrm{~A} \& 1 \mathrm{E}$ of the annual Accountability Report. 
FINAL - JUNE 2016

\section{UNIVERSITY TUITION, FEES AND HOUSING PROJECTIONS}

University: USF Sarasota-Manatee

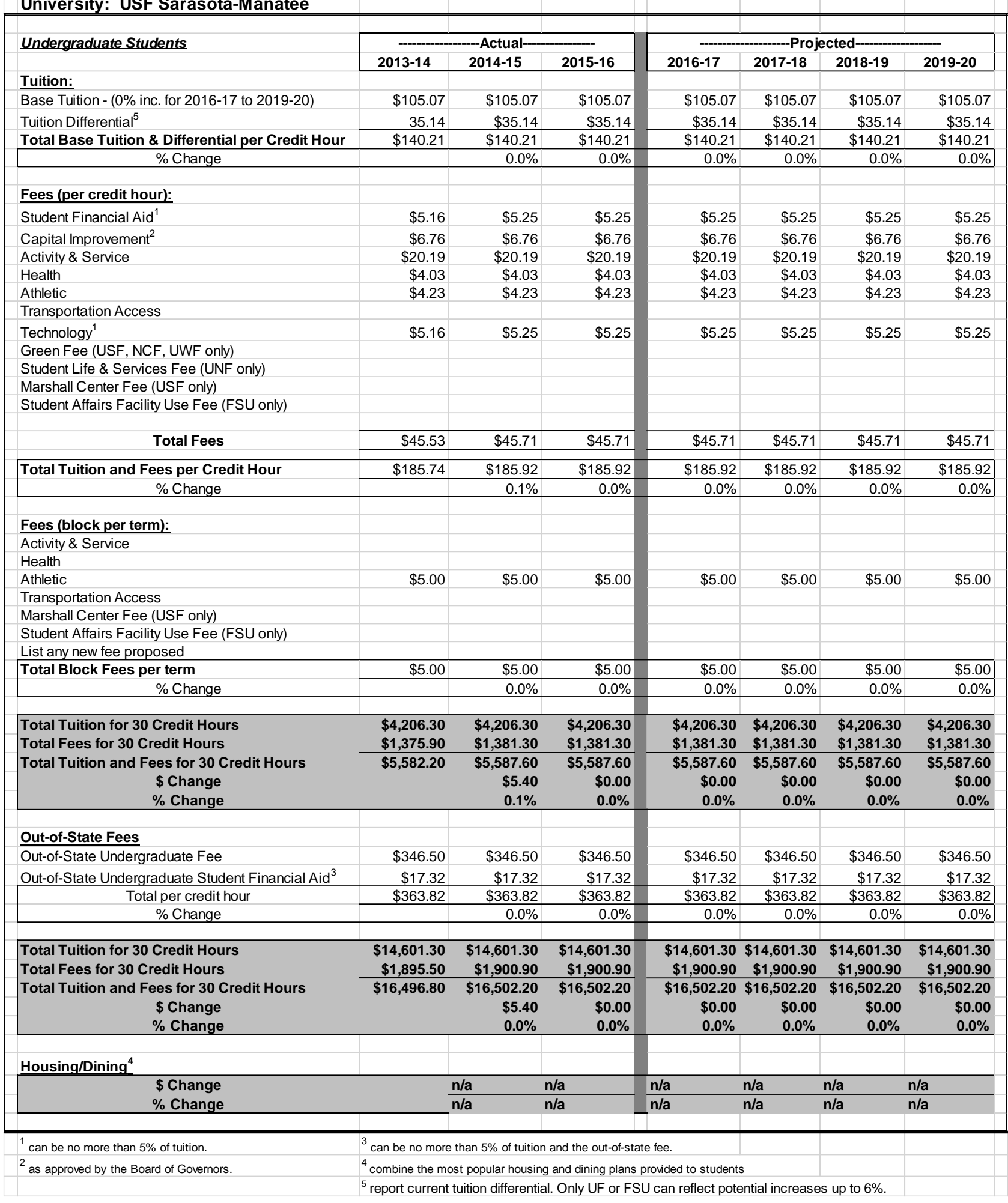

Note: The University will continually assess the need for changes to tuition \& fees in support of student success and fiscal responsibility consistent with FL Statute 1009.24 
FINAL - JUNE 2016

\section{DEFINITIONS}

\section{Performance Based Funding}

Percent of Bachelor's Graduates Enrolled or Employed (\$25,000+) in the U.S. One Year After Graduation

\section{Median Wages \\ of Bachelor's Graduates Employed Full-time in Florida One Year After Graduation}

This metric is based on the percentage of a graduating class of bachelor's degree recipients who are enrolled or employed (earning at least $\$ 25,000$ ) somewhere in the United States. Students who do not have valid social security numbers and are not found enrolled are excluded. Note: This data now non-Florida employment data.

Sources: State University Database System (SUDS), Florida Education \& Training Placement Information Program (FETPIP) analysis of Wage Record Interchange System (WRIS2) and Federal Employment Data Exchange (FEDES), and National Student Clearinghouse (NSC).
This metric is based on annualized Unemployment Insurance (UI) wage data from the fourth fiscal quarter after graduation for bachelor's recipients. UI wage data does not include individuals who are self-employed, employed out of state, employed by the military or federal government, those without a valid social security number, or making less than minimum wage. Sources: State University Database System (SUDS), Florida Education \& Training Placement Information Program (FETPIP), National Student Clearinghouse.

For each of the last four years of data, the annual undergraduate total full expenditures (includes direct and indirect expenditures) were divided by the total fundable student credit hours to create a cost per credit hour for each year. This cost per credit hour was then multiplied by 30 credit hours to derive an average annual cost. The average annual cost for each of the four years was summed to provide an average cost per degree for a baccalaureate degree that requires 120 credit hours. Sources: State University Database System (SUDS), Expenditure Analysis: Report IV.

Six Year FTIC Graduation Rate
This metric is based on the percentage of first-time-in-college (FTIC) students who started in the Fall (or summer continuing to Fall) term and had graduated from the same institution within six years. Source: Accountability Report (Table 4D).

\section{Academic \\ Progress Rate \\ 2nd Year Retention \\ with GPA Above 2.0}

This metric is based on the percentage of first-time-in-college (FTIC) students who started in the Fall (or summer continuing to Fall) term and were enrolled full-time in their first semester and were still enrolled in the same institution during the Fall term following their first year with had a grade point average (GPA) of at least 2.0 at the end of their first year (Fall, Spring, Summer). Source: Accountability Report (Table 4B).

\section{Bachelor's Degrees within Programs of Strategic Emphasis}

This metric is based on the number of baccalaureate degrees awarded within the programs designated by the Board of Governors as 'Programs of Strategic Emphasis'. A student who has multiple majors in the subset of targeted Classification of Instruction Program codes will be counted twice (i.e., double-majors are included). Source: Accountability Report (Table $4 \mathrm{H})$.

This metric is based the number of undergraduates, enrolled during the fall term, who received a Pell-grant during the fall term. Unclassified students, who are not eligible for Pellgrants, were excluded from this metric. Source: Accountability Report (Table 3E).

This metric is based on the number of graduate degrees awarded within the programs designated by the Board of Governors as 'Programs of Strategic Emphasis'. A student who has multiple majors in the subset of targeted Classification of Instruction Program codes will be counted twice (i.e., double-majors are included). Source: Accountability Report (Table $5 \mathrm{C})$. 
FINAL - JUNE 2016

\section{BOG Choice Metrics}

\section{Percent of Bachelor's Degrees Without Excess Hours}

This metric is based on the percentage of baccalaureate degrees awarded within $110 \%$ of the credit hours required for a degree based on the Board of Governors Academic Program Inventory.

Note: It is important to note that the statutory provisions of the "Excess Hour Surcharge" (1009.286, FS) have been modified several times by the Florida Legislature, resulting in a phased-in approach that has created three different cohorts of students with different requirements. The performance funding metric data is based on the latest statutory requirements that mandates $110 \%$ of required hours as the threshold. In accordance with statute, this metric excludes the following types of student credits (ie, accelerated mechanisms, remedial coursework, non-native credit hours that are not used toward the degree, non-native credit hours from failed, incomplete, withdrawn, or repeated courses, credit hours from internship programs, credit hours up to 10 foreign language credit hours, and credit hours earned in military science courses that are part of the Reserve Officers' Training Corps (ROTC) program). Source: State University Database System (SUDS).

\section{BOT Choice Metrics}

Number of Postdoctoral
Appointees
USF

This metric is based on the number of post-doctoral appointees at the beginning of the academic year. A postdoctoral researcher has recently earned a doctoral (or foreign equivalent) degree and has a temporary paid appointment to focus on specialized research/scholarship under the supervision of a senior scholar.

Source: National Science Foundation/National Institutes of Health annual Survey of Graduate Students and Postdoctorates in Science and Engineering (GSS).

\section{Key Performance Indicators \\ Teaching \& Learning Metrics}

Freshmen in Top $10 \%$

of HS Graduating Class

Professional/Licensure Exam First-time Pass Rates

Average Time to Degree Mean Years for FTIC in $120 \mathrm{hr}$ programs

\section{FTIC Graduation Rates In 4 years (or less)}

\section{Bachelor's Degrees Awarded}

Graduate Degrees Awarded
Percent of all degree-seeking, first-time, first-year (freshman) students who had high school class rank within the top $10 \%$ of their graduating high school class. As reported by the university to the Common Data Set (C10).

The number of exams with first-time pass rates above and below the national or state average, as reported in the annual Accountability report, including: Nursing, Law, Medicine (3 subtests), Veterinary, Pharmacy, Dental (2 subtests), Physical Therapy, and Occupational Therapy.

This metric is the mean number of years between the start date (using date of most recent admission) and the end date (using the last month in the term degree was granted) for a graduating class of first-time, single-major baccalaureates in 120 credit hour programs within a (Summer, Fall, Spring) year.

As reported in the annual Accountability report (table 4D), First-time-in-college (FTIC) cohort is defined as undergraduates entering in fall term (or summer continuing to fall) with fewer than 12 hours earned since high school graduation. The rate is the percentage of the initial cohort that has either graduated from or is still enrolled in the same institution by the fourth academic year. Both full-time and part-time students are used in the calculation. The initial cohort is revised to remove students, who have allowable exclusions as defined by IPEDS, from the cohort.

This is a count of baccalaureate degrees awarded as reported in the annual Accountability Report (Table 4G).

This is a count of graduate degrees awarded as reported in the Accountability Report (Table $5 B)$. 
Bachelor's Degrees Awarded To African-American and Hispanic Students

Adult (Aged 25+) Undergraduates Enrolled Fall term

Percent of Undergraduate FTE Enrolled in Online Courses

Percent of Bachelor's Degrees in STEM \& Health

Percent of Graduate Degrees in STEM \& Health
Non-Hispanic Black and Hispanic do not include students classified as Non-Resident Alien or students with a missing race code - as reported in the Accountability Report (table 4I).

Students who earn two distinct degrees in the same term are counted twice - whether their degrees are from the same six-digit CIP code or different CIP codes. Students who earn only one degree are counted once - even if they completed multiple majors or tracks. Percentage of Degrees is based on the number of baccalaureate degrees awarded to non-Hispanic Black and Hispanic students divided by the total degrees awarded - excluding those awarded to non-resident aliens and unreported.

This metric is based on the age of the student at the time of enrollment (not upon entry). Age acts as a surrogate variable that captures a large, heterogeneous population of adult students who often have family and work responsibilities as well as other life circumstances that can interfere with successful completion of educational objectives.

Full-time Equivalent (FTE) student is a measure of instructional activity that is based on the number of credit hours that students enroll. FTE is based on the US definition, which divides undergraduate credit hours by 30 . Distance Learning is a course in which at least 80 percent of the direct instruction of the course is delivered using some form of technology when the student and instructor are separated by time or space, or both (per 1009.24(17), F.S.). The percentage of baccalaureate degrees that are classified as STEM by the Board of Governors in the SUS program inventory as reported in the annual Accountability Report (Table 4H).

The percentage of baccalaureate degrees that are classified as STEM by the Board of Governors in the SUS program inventory as reported in the annual Accountability Report (Table 5C). 


\section{Student Debt Summary}

\section{Percent of Bachelor's Recipients with Debt}

This is the percentage of bachelor's graduates in a given academic year who entered the university as a first-time-in-college (FTIC) student and who borrowed through any loan programs (institutional, state, Federal Perkins, Federal Stafford Subsidized and unsubsidized, private) that were certified by your institution - excludes parent loans. Source: Common Dataset (H4).

This is the average amount of cumulative principal borrowed (from any loan program certified by the institution) for each native, FTIC bachelor's recipient in a given academic year that graduated with debt - see metric definition above. This average does NOT include students who did not enter a loan program that was certified by the institution. Source: Common Dataset (H5).

Student loan cohort default rate (CDR) data includes undergraduate and graduate students, and refers to the three federal fiscal year period when the borrower enters repayment and ends on the second fiscal year following the fiscal year in which the borrower entered repayment. Cohort default rates are based on the number of borrowers who enter repayment, not the number and type of loans that enter repayment. A borrower with multiple loans from the same school whose loans enter repayment during the same cohort fiscal year will be included in the formula only once for that cohort fiscal year. Default rate debt includes: Federal Stafford Loans, and Direct Stafford/Ford Loans - for more information see: http://ifap.ed.gov/DefaultManagement/CDRGuideMaster.html.

\begin{tabular}{|c|c|c|c|}
\hline \multicolumn{4}{|c|}{ Three Year CDR } \\
\hline $\begin{array}{l}\text { Cohort } \\
\text { Fiscal } \\
\text { Year }\end{array}$ & $\begin{array}{c}\text { Year } \\
\text { Published }\end{array}$ & $\begin{array}{l}\text { Borrowers in the Numerator } \\
\text { Borrowers in the Denominator }\end{array}$ & $\begin{array}{l}\frac{\text { 3-Yr Time Period }}{\text { (Numerator) }} \\
\text { 1-Yr Time Period } \\
\text { (Denominator) }\end{array}$ \\
\hline 2009 & 2012 & $\begin{array}{l}\text { Borrowers who entered repayment in } 2009 \\
\text { and defaulted in } 2009,2010 \text { or } 2011 \\
\text { Borrowers who entered repayment in } 2009\end{array}$ & $\frac{10 / 01 / 2008 \text { to } 9 / 30 / 2011}{10 / 01 / 2008 \text { to } 9 / 30 / 2009}$ \\
\hline 2010 & 2013 & $\begin{array}{l}\text { Borrowers who entered repayment in } 2010 \\
\text { and defaulted in } 2010,2011 \text { or } 2012 \\
\text { Borrowers who entered repayment in } 2010\end{array}$ & $\frac{10 / 01 / 2009 \text { to } 9 / 30 / 2012}{10 / 01 / 2009 \text { to } 9 / 30 / 2010}$ \\
\hline 2011 & $2014^{*}$ & $\begin{array}{l}\text { Borrowers who entered repayment in } 2011 \\
\text { and defaulted in } 2011,2012 \text { or } 2013 \\
\text { Borrowers who entered repayment in } 2011\end{array}$ & $\frac{10 / 01 / 2010 \text { to } 9 / 30 / 2013}{10 / 01 / 2010 \text { to } 9 / 30 / 2011}$ \\
\hline 2012 & 2015 & $\begin{array}{l}\text { Borrowers who entered repayment in } 2012 \\
\text { and defaulted in } 2012,2013 \text { or } 2014 \\
\text { Borrowers who entered repayment in } 2012\end{array}$ & $\frac{10 / 01 / 2011 \text { to } 9 / 30 / 2014}{10 / 01 / 2011 \text { to } 9 / 30 / 2012}$ \\
\hline 2013 & 2016 & $\begin{array}{l}\text { Borrowers who entered repayment in } 2013 \\
\text { and defaulted in } 2013,2014 \text { or } 2015 \\
\text { Borrowers who entered repayment in } 2013\end{array}$ & $\frac{10 / 01 / 2012 \text { to } 9 / 30 / 2015}{10 / 01 / 2012 \text { to } 9 / 30 / 2013}$ \\
\hline 2014 & 2017 & $\begin{array}{l}\text { Borrowers who entered repayment in } 2014 \\
\text { and defaulted in } 2014,2015 \text { or } 2016 \\
\text { Borrowers who entered repayment in } 2014\end{array}$ & $\frac{10 / 01 / 2013 \text { to } 9 / 30 / 2016}{10 / 01 / 2013 \text { to } 9 / 30 / 2014}$ \\
\hline 2015 & 2018 & $\begin{array}{l}\text { Borrowers who entered repayment in } 2015 \\
\text { and defaulted in } 2015,2016 \text { or } 2017 \\
\text { Borrowers who entered repayment in } 2015\end{array}$ & $\frac{10 / 01 / 2014 \text { to } 9 / 30 / 2017}{10 / 01 / 2014 \text { to } 9 / 30 / 2015}$ \\
\hline
\end{tabular}

